\title{
Expression of NSE and AFI in Fatal Lamb's Lungs before and after Relieving Ureteral Obstruction
}

\author{
Gaoyan Deng' ${ }^{1 \#}$ Fenghua Wang ${ }^{1}$ Junjie Sun², Jiacong $\mathrm{Mo}^{2}$, \\ Zhijian Deng ${ }^{1}$, Wei Liu ${ }^{1}$ \\ ${ }^{1}$ Department of Pediatric Surgery, Guangzhou Women and Children's Medical Center, Guangzhou, China \\ ${ }^{2}$ Department of Pediatric Surgery, The First Affiliated Hospital of Sun Yat-sen University, Guangzhou, China \\ Email: \#denggaoyanemail@163.com
}

Received April 23, 2011; revised May 5, 2012; accepted May 17, 2012

\begin{abstract}
Aims: To evaluate the Expression of NSE and AFI before and after relieving ureteral obstruction in fatal lamb's lungs with unilateral hydronephrosis. Study Design and Subjects: Forty-five pregnant lambs were randomly divided into 3 groups. Eighteen lambs were in the hydronephrotic model group. The second group included 9 lambs in which ureteral obstruction was relieved 3 weeks after the initial operation. Eighteen lambs were in the control group which was just performed open hysterotomies. Outcome measures: The amniotic fluid index (AFI) and the expression of neuronspecific enolase (NSE) in the lungs were tested. Results: In the hydronephrotic model group, the AFI was less and the expression of NSE was greater than the control group. The AFI and expression of NSE were similar to the control group after relieving the ureteral obstruction. Conclusions: The AFI and expression of NSE return to normal after after relieving the ureteral obstruction, which meant that relieving ureteral obstruction is perhaps beneficial to lung development.
\end{abstract}

Keywords: Hydronephrosis; Neuron Specific Enolase; Fetus; Lamb

\section{Introduction}

Ureteropelvic junction obstruction (UPJO) is a common cause of fetal hydronephrosis; the incidence of UPJO is 1 in 2000 [1]. At present, infants with UPJO are managed expectantly or undergo surgical treatment based on the grade of hydronephrosis or renal function. Wang [2] reported that relief of ureteral obstruction in utero may prevent or attenuate the development of nephropathy in lambs. In late gestation the amniotic fluid, which benefits lung development, is primarily derived from the kidneys. Harrison [3] and Crombleholme [4] concluded that lung function benefits from relief of ureteral obstruction in utero; however, the studies had no objective indicator to monitor lung development.

Neuron-specific enolase (NSE), which is secreted by neuron and neuroendorine cells, is a crucial enzyme for glucolysis. During lung development in lambs, NSEpositive cells exist beginning at 5 weeks of gestation. Thus, NSE is an indicator by which lung development can be monitored [5-7]. The expression of NSE in fetal lungs with ureteral obstruction has been the subject of a

\footnotetext{
*Supported by the Grants 2007B030502008 from Hall of Science and Technology, Guangdong Province, China.

"Corresponding author.
}

study [8]. In the current study, the effect of changes in amniotic fluid and expression of NSE in lungs before and after relief of UPJO in utero on lung development were determined.

\section{Materials and Methods}

\subsection{Animals}

Forty-five healthy Bore lambs with singleton gestations, 1 - 1.5 years old and weighing $17.5 \pm 2.2 \mathrm{~kg}$ (range, 14 $22 \mathrm{~kg}$ ), were used in the current experiments. All of the lambs were pregnant when obtained from the Bore lambs basement in Zeng Cheng City of Guang Dong Province in China. The lambs were randomly divided into three groups. Eighteen lambs were in the control group; 14 fetal lungs were obtained antepartum and 4 fetal lungs were obtained postpartum. In the hydronephrotic model group, 14 of 18 fetal lungs were obtained antepartum (7 cases within 1 week and 7 cases within 3 weeks after ureteral obstruction) and 4 fetal lungs were obtained postpartum. The third group included 9 lambs in which the obstruction was relieved 3 weeks after the initial operation; 4 fetal lungs were obtained antepartum and 5 fetal lungs were obtained postpartum. 


\subsection{Operations}

Open hysterotomies were performed on pregnant ewes via flank laparotomies, and the fetal lamb hindquarters were then exteriorized. After exposing the upper part of the left ureter in the fetuses, an F6 split silastic tube was wrapped around the ureter to effect obstruction. Methylthionium chloride ( $2 \mathrm{~mL}$ ) was injected into the renal pelvis to demonstrate whether or not it could pass through the obstructed portion of the ureter. We repositioned the fetuses and closed the uterine cavity as well as the abdominal wall.

Three weeks later, another operation was performed to remove the obstruction (the silastic tubes) in 9 lambs. All protocols were approved by Medical Ethics Committee of the First Affiliated Hospital of Sun Tat-Sen University.

\subsection{Tests}

An ultrasound was performed pre-operatively and when the lungs were obtained. The amniotic fluid index (AFI) was estimated by the sum of depth of four amniotic quadrants.

The lung tissues of the lambs were processed for immunohistochemical staining using the S-P immunohistochemical method. Expression of NSE was detected using mouse polyclonal anti-NSE antibody (No: MAB-0584, Colonial Number: E27, 1:200; Maxin Biotechnology Company Limited, Fu Zhou of Fu Jian Province, China). Cells with brown staining were considered positive. One hundred epithelial cells were tested randomly under microscopy; the calculation of positive cells was analyzed.

\subsection{Statistical Analysis}

The information obtained was entered into computer software and analyzed using SPSS progrmme version 13 (SPSS, Inc., Chicago, IL, USA). The results were expressed as the means \pm SD. A t-test was used to test the association of the Expression of NSE and AFI before and after relieve of ureteral obstruction. The results were considered significant when $\mathrm{P}<0.005$.

\section{Results}

There were two types of lung tissues visualized under microscopy. One type was in the tubular period, and showed multiple tubular structures (Figure 1). Another type of lung tissue was composed of an alveolar structure in the alveolar period (Figure 2), which had deplanatetype I epithelium and cubic-type II epithelium. According to a histologic study, the lungs obtained within 1 week of the initial operation were in the tubular period, while 3 weeks later the lungs were in the alveolar period. Immunohistochemical staining showed that the plasma of

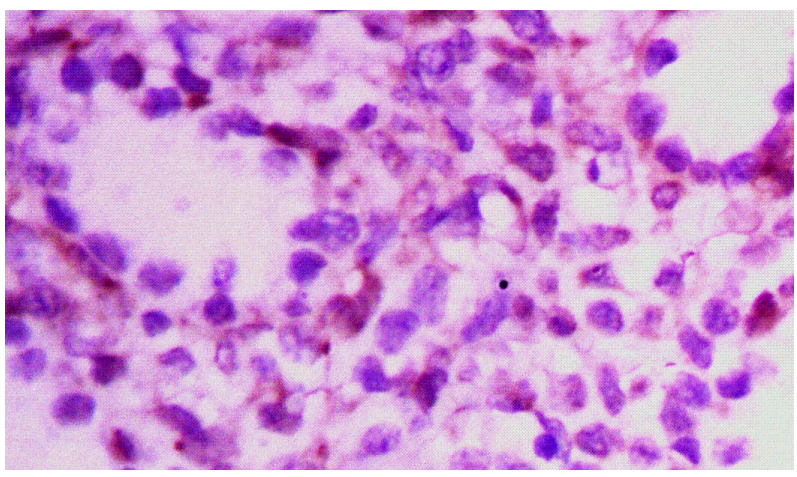

Figure 1. Pulmonary tissue in tubular period.

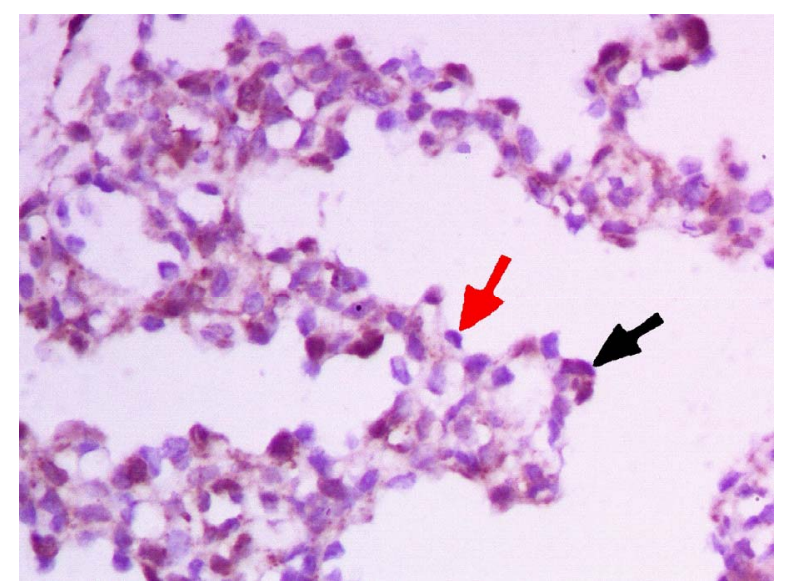

Figure 2. Pulmonary tissue in alveolar period, black arrow shows a NSE positive cell, red arrow shows a NSE negative cell.

positive cells was brown with a blue nucleus (shown by the black arrow in Figure 2). The plasma of negative cells was transparent (shown by the red arrow in Figure 2). Moreover, some smooth muscular cells and vascular epithelium in the interstitial tissue were NSE-positive.

In the hydronephrotic group, the AFI in lungs within 1 week after obstruction (in the tubular period) had no significant change compared with the control group $(\mathrm{P}=$ 0.55, Table 1). The AFI in lungs 3 weeks after obstruction (in the alveolar period) and postpartum were less than the control group ( $\mathrm{P}=0.02$ and $\mathrm{P}=0.01$, respectively). In the group in which obstruction was relieved, the AFI in lungs 3 weeks after obstruction (in the alveolar period) was greater than the hydronephrotic group ( $\mathrm{P}$ $=0.03)$, but not different $(P=0.3)$ compared with the control group. Postpartum, the AFI in lungs was greater than the hydronephrotic group $(\mathrm{P}=0.02)$, but not different $(P=0.4)$ compared with the control group.

In the control group, the number of NSE-positive cells in the alveolar period was less than the tubular period ( $\mathrm{P}$ $=0.02$, Table 2), and was not different postpartum $(\mathrm{P}=$ 0.22 ). In the hydronephrotic group, the number of NSEpositive cells in the lungs within 1 week after obstruction 
Table 1. AFI in three groups.

\begin{tabular}{cccc}
\hline & tubular period & alveolar period & after birth \\
\hline normal & $15.6 \pm 1.7$ & $13.1 \pm 1.4$ & $9.5 \pm 1.3$ \\
hydronephrosisi & $14.5 \pm 2.1$ & $6.7 \pm 1.8$ & $6.7 \pm 1.8$ \\
P & 0.55 & 0.02 & 0.01 \\
relieving obstruction & & $11.5 \pm 1.3$ & $8.2 \pm 1.3$ \\
P & & 0.3 & 0.4 \\
\hline
\end{tabular}

Table 2. NSE expression in three group*"

\begin{tabular}{cccc}
\hline & tubular period & alveolar period & after birth \\
\hline normal & $37.6 \pm 1.4$ & $29.7 \pm 2.5$ & $28 \pm 1.4$ \\
hydronephrosisi & $37.1 \pm 1.4$ & $33.1 \pm 2.6$ & $35.6 \pm 1.2$ \\
P & 0.65 & 0.001 & 0.002 \\
relieving obstruction & & $26.8 \pm 1.9$ & $29.2 \pm 3.4$ \\
P & & & 0.56 \\
\hline
\end{tabular}

* positive cells in forty multiple microscopy per 100 alveolar epithelial cells.

(in the tubular period) had no changes compared with the control group $(\mathrm{P}=0.65)$. Three weeks later (in the alveolar period), the number of NSE-positive cells were greater than the control group $(\mathrm{P}=0.001)$. Postpartum, the number of NSE-positive cells were greater than the control group $(\mathrm{P}=0.002)$. In the group in which ureteral obstruction was relieved, the number of NSE-positive cells in the lungs after birth were less than the hydronephrotic group ( $\mathrm{P}=0.02)$, having no difference with the control group $(\mathrm{P}=0.56)$.

\section{Discussion}

In our study, the lambs were divided into three groups and analyzed in three different periods (tubular, alveolar, and postpartum periods). According to Alcorn's criteria [9], lung development in the current study was in the tubular and alveolar periods. According to the histologic studies under microscopy, when ureteral obstruction was performed the lungs in the lambs were in the tubular period (1 week later). When the obstruction was relieved 3 weeks later, the lungs were in the alveolar period. Postpartum, the lungs were also in the alveolar period. There were some NSE-positive smooth muscular cells and vascular epithelium in the interstitial tissue because the NSE antibody could stain these tissues.

The current study showed that amniotic fluid did not change significantly within 1 week after hydronephrosis, suggesting that the volume of amniotic fluid could be normal in a short time with only one kidney; however, the amniotic fluid was $<3$ weeks later. Moreover, the amniotic fluid was more turbid in the hydronephrotic group in the current study. Though the lamb neonates had no respiratory embarrassment, the amniotic fluid was less than the control group. As shown in the current study, after the obstruction was relieved, the amniotic fluid increased. Thus, we know that amniotic fluid is beneficial to lung development. The results of our study suggest that relieving ureteral obstruction in utero is helpful for lung development.

The expression of NSE in the lungs in the hydronephrotic group was more than the control group in this study, suggesting that lung development could be impaired in the lamb fetus with unilateral hydronephrosis. Lung development in the lambs with only one kidney was worse than normal, although they had no respiratory embarrassment because a lamb without respiratory distress at birth does not mean that it has no lung impairment. The expression of NSE returned to normal after relieving the ureteral obstruction, which suggested that lung dysgenesis can be reversed by relieving ureteral obstruction in utero. This finding would encourage physicians to relieve ureteral obstruction in utero, not only for the probability of recovery of the kidneys, but also for lung development.

Because the expression of NSE differs in different anatomic locations [5], it is preferable to analyze the expression of NSE in different anatomic locations to reach a more comprehensive conclusion. As the lung tissues of our study were obtained from a peripheral location, we believe that lung development would benefit from relieving ureteral obstruction. The AFI and expression of NSE return to normal after relieving the ureteral obstruction, which meant that relieving ureteral obstruction is perhaps beneficial to lung development.

\section{REFERENCES}

[1] A. Becker and M. Baum, “Obstructive Uropathy,” Early Human Development, Vol. 82, No. 1, 2006, pp. 15-22. doi:10.1016/j.earlhumdev.2005.11.002

[2] F. H. Wang, J. J. Sun, G. Y. Deng and J. C. Mo, "Does Intervention in Utero Preserve the Obstructed Kidneys of Fetal Lambs? A Histological, Cytological, and Molecular Study," Pediatric Search, Vol. 66, No. 2, 2009, pp. 145148.

[3] M. R. Harrison, D. K. Nakayama, R. Noall and A. A. de Lorimier, "Correction of Congenital Hydronephrosis in Utero II, Decompression Reverses the Effects of Obstruction on the Fetal Lung and Urinary Tract," Journal of Pediatric Surgery, Vol. 17, No. 6, 1982, pp. 965-974. doi:10.1016/S0022-3468(82)80476-4

[4] T. M. Crombleholme, M. R. Harrison, M. S. Golbus, M. T. Longaker, J. C. Langer, P. W. Callen, R. L. Anderson, R. B. Goldstein and R. A. Filly, "Fetal Intervention in Obstructive Uropathy: Prognostic Indicators and Efficacy of Intervention," American Journal of Obstetrics and Gynecology, Vol. 162, No. 5, 1990, pp. 1239-1244.

[5] M. N. Sheppard, P. J. Marangos, S. R. Bloom and J. M. Polak, "Neuron Specific Enolase: A Marker for the Early Development of Nerves and Endocrine Cells in the $\mathrm{Hu}-$ 
man Lung,” Life Science, Vol. 34, No. 3, 1984, pp. 265271. doi:10.1016/0024-3205(84)90598-8

[6] K. Asabe, R. W. Jennings, M. R. Harrison and S. Suita, "Quantitative Study of Pulmonary Endocrine Cells in Fetal, Postnatal and Adult Lambs," Journal of Veterinary Medical Science, Vol. 66, No. 4, 2004, pp. 373-380. doi:10.1292/jvms.66.373

[7] L. Balaguer and J. Romano, "Solitary Neuroendorine Cells and Neuroepithelial Bodies in the Lower Airways of
Embryonic, Fetal, and Postnatal Lambs,” The Anatomical Record, Vol. 231, No. 3, 1991, pp. 333-338. doi:10.1002/ar.1092310306

[8] D. G. Alcorn, T. M. Adamson, J. E. Maloney and P. M. Robinson, "A Morphologic and Morphometric Analysis of Fetal Lung Development in the Lambs," Anatomy Research, Vol. 201, No. 4, 1981, pp. 655-667. doi:10.1002/ar.1092010410 